\title{
$N$ of 1 trials in diabetes: making individual therapeutic decisions
}

\author{
A. Tsapas • D. R. Matthews
}

Received: 18 February 2008 / Accepted: 21 February 2008/Published online: 4 April 2008

(C) Springer-Verlag 2008

\begin{abstract}
N$ of 1 trials are single-subject, randomised, crossover studies, in which the patient serves as their own control to compare the efficacy of a treatment. They offer an alternative to large, randomised clinical trials, which have limited applicability, and empirical testing, which is arbitrary in nature. $N$ of 1 trials are regarded as providing the highest strength of evidence for individual subject decisions. They rely upon the patient expressing preferences and making shared decisions for determining the future therapy, and may therefore lead to the successful implementation of lifestyle interventions. $N$ of 1 trials are an optimal approach for making therapeutic decisions in chronic diseases like diabetes mellitus, where decision-making is often reliant upon arbitrary criteria and clinical judgement.
\end{abstract}

Keywords Diabetes $\cdot N$ of $1 \cdot$ Therapeutic decisions $\cdot$ Trials

\section{A. Tsapas}

Second Medical Department, Aristotle University,

Thessaloniki, Greece

\author{
A. Tsapas $\cdot$ D. R. Matthews \\ Oxford Centre for Diabetes, Endocrinology and Metabolism, \\ University of Oxford, \\ Oxford, UK
}

\author{
A. Tsapas $(\square)$ \\ Oxford Centre for Diabetes, Endocrinology and Metabolism, \\ Churchill Hospital, \\ Headington, \\ Oxford OX3 7LJ, UK \\ e-mail: atsapas@auth.gr
}

\begin{abstract}
Abbreviation
RCT Randomised clinical trial
\end{abstract}

\section{Introduction}

The publication of data linking a lack of glycaemic control to micro- and macrovascular complications and increased mortality, in both type 1 [1, 2] and type 2 diabetes [3], has led to the establishment of a goal-driven approach to decision-making in diabetes: guidelines and consensus statements issued by several organisations $[4,5]$ advise that tight glycaemic control should be strived for, often recommending a range of glucose values limited to the extent that they might negatively affect the quality of life [6] or even the mortality of individuals with diabetes [7]. The guidelines and consensus statements also suggest therapeutic pathways, aiding decisions with respect to choice of treatment [5]. Unfortunately however, owing to limitations already acknowledged by most of them (paucity of high-quality evidence, diversity of clinical characteristics, progressive nature of the disease), they propose algorithms that are based upon 'clinical judgement and experience', which have long been criticised for being unreliable [8].

\section{Decision-making in diabetes in the modern era}

The problems associated with treatment decision-making in chronic diseases are familiar to those in everyday clinical practice [9]: several goals to be met, multiple strategies for 
achieving them, and multiple options available for each strategy. Consequently, healthcare professionals, even when evaluating the same patient, make completely different treatment choices [10], influenced by multiple factors such as subjective attitudes and ideas, experience, regional prescribing habits, cost and availability of medications, and the patient's stated or perceived preferences [11]. These inconsistencies with respect to treatment highlight the need to shift focus and engage the patient in the decision-making process, a strategy that can prove particularly effective in diabetes, a chronic disease for which successful management necessitates sustained lifestyle interventions and aggressive multifactorial management of several risk factors $[11,12]$. Patient involvement does not, however, imply that we should move away from evidence-based medicine. On the contrary, we should be using current evidence of efficacy to allow patients to make an informed choice between therapies, bearing in mind that an individual's response to any regimen has aspects of uncertainty.

\section{Evidence-based medicine and clinical practice}

Evidence-based medicine is defined as 'the conscientious, explicit and judicious use of current best evidence in making decisions about the care of the individual patient' [13]. Unfortunately, however, 'evidence-based medicine' is often misunderstood as meaning 'randomised clinical trials (RCTs)based medicine', despite the fact that RCTs are associated with certain limitations regarding their applicability and validity [14]. To reduce variability in the reported results, researchers may try to recruit a population that is as homogeneous as possible, by applying restrictive inclusion and exclusion criteria. This, however, results in trial participants being recruited from just a minority of the patients one sees in standard clinical care, and so the findings may not be applicable to the majority of patients. The use of a broader recruitment strategy, increasing the heterogeneity of a trial's population (and thus the applicability of the results) may lead to wider confidence intervals. Moreover, parameters are not monitored as meticulously in clinical practice as they are within the setting of a clinical trial. Finally, there is always a significant time lag between the development of a new therapeutic modality and the emergence of sufficient and robust evidence-based data.

In situations where evidence-based data are lacking, healthcare professionals often use a trial of practice, also called empirical testing. These may be specific therapeutic decisions, based on consensus or treatment guidelines, but they are, nevertheless, based on arbitrary criteria. A therapy may have an excellent evidence base for its use but there may not be any consistent evidence available for its use in preference to other therapeutic options. Thus, in case history terms, it is debatable a

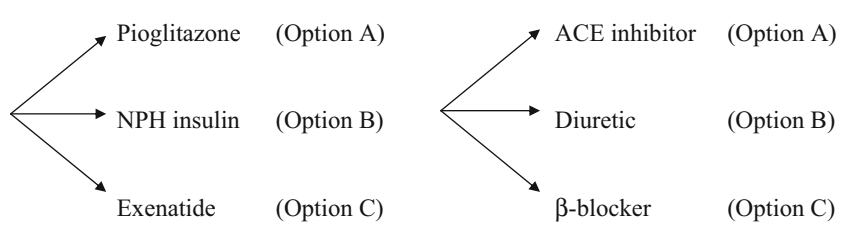

Fig. 1 a Decision tree for treatment of hyperglycaemia in a patient with type 2 diabetes. b Decision tree for treatment of hypertension. The options for therapy have an evidence base for their individual effects, but in any given patient the choice between option A, B or C is arbitrary

as to which agent should be added to metformin and sulfonylurea therapy [10]. Pioglitazone, NPH insulin and exenatide all have excellent evidence bases for their use. However, there are no data available that might indicate which choice a physician should make for a specific patient (Fig. 1). The New England Journal of Medicine has illustrated this by publishing the results of a worldwide opinion poll showing national biases and subjective physician preferences [11].

Having made a therapeutic decision, a physician is unlikely to later change this. A patient might have responded much better to exenatide than to a glitazone (or vice versa), but this will remain untested. Moreover, the therapeutic benefits will be judged solely by the same physician, using no predefined criteria. There are other issues, often beyond voluntary control (placebo effect, the patient's wish to please his doctor, patient's/doctor's attitude towards the specific therapy), that have an impact upon the outcome, possibly leading to false confidence in an initial therapeutic decision [15].

\section{$N$ of 1 trials and quality of evidence}

$N$ of 1 trials have been proposed as an alternative to these problems [16]. $N$ of 1 trials are single-subject, randomised, crossover (double-blinded where possible) studies in which the patient serves as their own control to compare the efficacy of a treatment vs placebo or two treatments. Unlike crossover studies, $N$ of 1 trials attempt to establish effects in an individual. They contain the same safeguards as RCTsrandomisation, definition and quantification of outcomes, and patient and physician blinding to treatment assignmentand are considered to offer robust evidence for making individual treatment decisions. In fact, as regards individual therapeutic decisions, the evidence offered by $N$ of 1 trials is considered to be of the highest strength, because its applicability and validity for the specific patient is higher than that of any RCT [14]. However, not every patient is eligible to participate in an $N$ of 1 trial, and there are strict criteria that apply to their design (see text box: Eligibility for an $N$ of 1 trial) [17]. 


\section{Eligibility for an $N$ of 1 trial}

1. Is an $n$ of 1 randomised trial indicated for this patient?

Is the effectiveness of the treatment option really in doubt?

Will the treatment, if effective, be continued in the long term?

Is the patient eager to collaborate in designing and carrying out an $n$ of 1 RCT?

2. Is an $n$ of 1 randomised trial feasible in this patient?

Does the treatment have an effect measurable over a limited time period?

Does the treatment cease to act soon after it is discontinued?

Is an optimal treatment duration feasible?

Can clinically relevant targets be measured?

Can sensible criteria for stopping the trial be established?

Should an unblinded run-in period be conducted?

3. Is an $n$ of 1 trial feasible in my practice setting?

Is there a pharmacist who can help me?

Are strategies for the interpretation of the trial data in place?

4. Is the study ethical?

$N$ of 1 trials are indicated in chronic diseases with a prolonged course. This is because it is not possible to test different therapeutic options over the time scale of an acute disease. Moreover, the interventions must have an effect that is readily observable, measurable and quantifiable over a limited time period. There should be a termination of effect, to avoid potential carry-over, which could interfere with the interpretation of data acquired from the sequential application of the interventions.

The use of $N$ of 1 trials has been suggested under various circumstances. They are an excellent option for the assessment of efficacy on an individual basis: they allow determination of magnitude of treatment effect, and therefore, titration of intervention or discontinuation of an ineffective treatment, and represent a cheap alternative to the wide application of pharmacogenomics. Under specific circumstances, they can also be used in the investigation of newly introduced therapies, to determine therapeutic range, side effects or target population. Perhaps even more important than measurable effects is the fact that the patients themselves can express an opinion as to which therapy they prefer, and the decision of long-term treatment can be made based solely on this. However, it is more likely that each arm of the trial will have a more complex outcome. A patient may prefer treatment $\mathrm{A}$, but treatment $\mathrm{B}$ may have a marginally better therapeutic effect, and treatment $\mathrm{C}$ may be cheaper than either A or B. The physician can then enter a constructive dialogue about future options, based on evidence and the patient's preference.

The methodology used in $N$ of 1 trials resembles that of crossover studies, except that it is implemented in single subjects. The patient sequentially undergoes each of the interventions to be examined (Fig. 2a). The acquisition of sufficient data together with the participant's safety and convenience are taken into consideration when determining the number and frequency of the treatment periods. To increase the consistency and the statistical power of the findings one could apply more periods of each regimen (Fig. 2b). The sequential application of different interventions may affect the findings as a result of possible

a

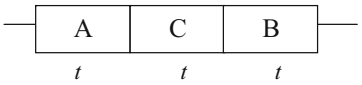

b

\begin{tabular}{|l|l|l|l|l|l|}
\hline $\mathrm{A}$ & $\mathrm{C}$ & $\mathrm{B}$ & $\mathrm{A}$ & $\mathrm{C}$ & $\mathrm{B}$ \\
\hline
\end{tabular}

Fig. 2 Simple randomisation schedule. A, B and C represent therapeutic regimens, as in Fig. 1. a $N$ of 1 trial with one treatment period for each agent. The length of $t$ should be sufficiently long to allow assessment of effect. b $N$ of 1 trial with two randomised treatment periods for each agent 
carry-over effects. This can be avoided by applying randomisation in longer blocks and by analysing data collected during the end of each period. Randomisation of intervention order is used to minimise systematic bias. To further reduce the observer-induced bias, the observer and the patient may be blinded to the intervention. However, it is not essential to the design of $N$ of 1 trials, as long as key variables and outcomes are recognised and predefined [18].

The main characteristic that differentiates $N$ of 1 trials from other trials, and may raise doubts about their clinical relevance and their scientific value, is their application to a single patient. The crucial point is that the results are not generalisable, but, rather, establishes the best therapy for an individual. Such a therapy will then be continued. This is in marked contrast to RCTs, which are designed to determine the efficacy of an agent in general, but may not be applicable to any single individual. Indeed, parametric statistical analysis recognises there is a large difference in therapeutic effect within a population, and RCTs tend to be large so that standard errors of a mean response are minimised. $N$ of 1 trials do not differ from other crossover studies in other respects. Outcome measures must be predefined, and the use of visual scales and symptom rating scales should be encouraged, to ensure that sufficient data from the patient regarding preferences is obtained. However, the use of statistical methods different from those used in the analysis of a classical crossover study may be warranted, to capture qualitative differences in predefined outcomes.

Can any valid conclusions be drawn by combining data from several similar $N$ of 1 trials, and if so, what is the appropriate methodology? Several methods for combining findings have been proposed, including the use of random effects models or hierarchical Bayesian models, based on a normal or binomial likelihood distribution. These methods are used to increase statistical power and offset any assumption of normality in the distribution of findings. They also overcome the inherent disadvantage of non-parametric statistics, which do not take magnitude into account when trying to rule-out chance. In this sense, the methodology used to analyse findings from a series of $N$ of 1 trials is similar to that used in meta-analyses.

\section{Ethical aspects}

Regarding the ethical aspects of $N$ of 1 trials, the requirements are similar to those of other trials: confidentiality, free and informed consent to participate, and withdrawal rights without any consequences on rights for care. However, since the objective of $N$ of 1 trials is to use the accumulated data and experience to formulate a treatment plan for a particular patient, the trial probably does not require formal ethical approval $[19,20]$. The trial in this case can be regarded as an audit of 1: an audit examines clinical practice, and the audit loop is completed when the results are utilised to change such practice for the future.

In summary, $N$ of 1 trials offer high-validity research in single subjects. They are easy and cheap to perform, and answer important questions for the specific patient. Indeed, for many patients the result of the trial will have ramifications for several years of future therapy, and this approach is regarded as providing the highest-grade evidence for making single-subject decisions.

\section{$N$ of 1 trials and patient involvement}

$N$ of 1 trials increase the patient's awareness and willingness to participate in the management of their disease [15, 21]. When assuming more responsibility for their healthcare, patients become more compliant and adhere better to their treatment regimen, they gain more knowledge and understanding of their disease and their treatment regimen and its relationship with their lifestyle, and they have an improved relationship with their healthcare professional. These facts may lead to better health-related outcomes [22], including increased patient satisfaction with care [23], shorter recovery periods [24] and improved general health [25]. Thus, careful assessment of the patient's preferred therapeutic regimen and a discussion of the objective outcomes is mandatory in $N$ of 1 trials. This discussion should be carefully undertaken and perhaps even formalised. The issues may be finely balanced between preference and outcome. An informed decision then needs to be made.

\section{$N$ of 1 trials in diabetes}

Although the use of $N$ of 1 trials in chronic disease research is rigorously advocated and there are robust data supporting their use, only one study on diabetes has been published to date, and this concerned the use of amitriptyline for the treatment of diabetic neuropathy [26]. Does this mean that $N$ of 1 trials are not indicated in diabetes?

Diabetes, both type 1 and type 2, fulfils many of the criteria required for a patient/disease to be eligible for study within an $N$ of 1 trial framework: it is a chronic disease and the effect of treatment (glycaemic control) is obvious soon after initiation and soon after discontinuation of therapy for most types of intervention. Most drugs do not have an extended carry-over effect on glucose (at least not so long as to bring into question the results of an $N$ of 1 trial). Moreover, the efficacy of interventions can be monitored thoroughly if multiple glucose readings are obtained, either with multiplepoints profiles with self-monitoring of blood glucose or with continuous glucose monitoring. Tailoring of treatment is a necessity for effective titration of diabetes therapy and 
elimination of treatment side effects. So it is remarkable that there has been just one trial of $N$ of 1 in diabetes.

\section{Conclusion}

$N$ of 1 trials offer a safe, objective and evidence-based way to implement patient-oriented research, individualisation of treatment, patient participation-all leading to improved outcomes and satisfaction-in everyday clinical practice of diabetes mellitus.

Acknowledgements A. Tsapas is supported by an Albert Renold Fellowship awarded by the European Foundation for the Study of Diabetes

Duality of interest The authors declare that there is no duality of interest associated with this manuscript.

\section{References}

1. The Diabetes Control and Complications Trial Research Group (1993) The effect of intensive treatment of diabetes on the development and progression of long-term complications in insulindependent diabetes mellitus. N Engl J Med 329:977-986

2. The Diabetes Control and Complications Trial/Epidemiology of Diabetes Interventions and Complications Research Group (2003) Intensive diabetes therapy and carotid intima-media thickness in type 1 diabetes mellitus. N Engl J Med 348:2294-2303

3. Stratton IM, Adler AI, Neil HA et al (2000) Association of glycaemia with macrovascular and microvascular complications of type 2 diabetes (UKPDS 35): prospective observational study. BMJ 321:405-412

4. American Diabetes Association (2008) Standards of medical care in diabetes-2008. Diabetes Care 31:S12-S54

5. Nathan DM, Buse JB, Davidson MB et al (2006) Management of hyperglycaemia in type 2 diabetes: a consensus algorithm for the initiation and adjustment of therapy. A consensus statement from the American Diabetes Association and the European Association for the Study of Diabetes. Diabetologia 49:1711-1721

6. The Diabetes Control and Complications Trial Research Group (1997) Hypoglycemia in the diabetes control and complications trial. Diabetes 46:271-286

7. National Institutes of Health (2008) For safety, NHLBI changes intensive blood sugar treatment strategy in clinical trial of diabetes and cardiovascular disease. Available from http://public.nhlbi.nih.
gov/newsroom/home/GetPressRelease.aspx?id=2551,accessed14/ $02 / 2008$

8. Anderson RM, Robins LS (1998) How do we know? Reflections on qualitative research in diabetes. Diabetes Care 21:1387-1388

9. Daar AS, Singer PA, Persad DL et al (2007) Grand challenges in chronic non-communicable diseases. Nature 450:494-496

10. Goldberg RB, Holman R, Drucker DJ (2008) Clinical decisions. Management of type 2 diabetes. N Engl J Med 358:293-297

11. Halperin F, Ingelfinger JR, McMahon GT (2008) Clinical decisions. Management of type 2 diabetes - polling results. N Engl J Med 358:e8

12. Gaede P, Lund-Andersen H, Parving H-H, Pedersen O (2008) Effect of a multifactorial intervention on mortality in type 2 diabetes. N Engl J Med 358:580-591

13. Sackett DL, Rosenberg WM, Gray JA, Haynes RB, Richardson WS (1996) Evidence based medicine: what it is and what it isn't. BMJ 312:71-72

14. Guyatt GH, Haynes RB, Jaeschke RZ et al (2000) Users' guides to the medical literature: XXV. Evidence-based medicine: principles for applying the users' guides to patient care. JAMA 284:1290-1296

15. Nikles CJ, Clavarino AM, Del Mar CB (2005) Using $n$-of-1 trials as a clinical tool to improve prescribing. Br J Gen Pract 55:175-180

16. Guyatt G, Sackett D, Adachi J et al (1988) A clinician's guide for conducting randomized trials in individual patients. CMAJ 139:497-503

17. Sackett DL, Havnes RB, Guyatt SH, Tugwell P (1991) Clinical epidemiology: a basic science for clinical medicine. Little, Brown \& Co., Boston

18. Brookes ST, Biddle L, Paterson C, Woolhead G, Dieppe P (2007) "Me's me and you's you" Exploring patients' perspectives of single patient ( $n$-of- 1 ) trials in the UK. Trials 8:10

19. Irwig L, Glasziou P, March L (1995) Ethics of $n$-of-1 trials. Lancet 345:469

20. Mahon JL, Feagan BG, Laupacis A (1995) Ethics of $n$-of- 1 trials. Lancet 345:989

21. Avins AL, Bent S, Neuhaus JM (2005) Use of an embedded Nof-1 trial to improve adherence and increase information from a clinical study. Contemp Clin Trials 26:397-401

22. Golin CE, DiMatteo MR, Gelberg L (1996) The role of patient participation in the doctor visit. Implications for adherence to diabetes care. Diabetes Care 19:1153-1164

23. Brody DS, Miller SM, Lerman CE, Smith DG, Caputo GC (1989) Patient perception of involvement in medical care: relationship to illness attitudes and outcomes. J Gen Intern Med 4:506-511

24. Ong LM, de Haes JC, Hoos AM, Lammes FB (1995) Doctor-patient communication: a review of the literature. Soc Sci Med 40:903-918

25. Stewart MA (1995) Effective physician-patient communication and health outcomes: a review. CMAJ 152:1423-1433

26. Hoogwerf BJ (1985) Amitriptyline treatment of painful diabetic neuropathy: an inadvertent single-patient clinical trial. Diabetes Care 8:526-527 